\title{
Topical Application of Ascorbic Acid Solution as an Adjunctive Method of Periodontal Therapy among Wet Snuff Users after Prosthodontics Therapy
}

\author{
Mohammed M. A. Abdullah Al-Abdaly ${ }^{1^{*}}$, Ali Saleh Alghamdi², Ahmad Hassan Alshehri \\ Faris Ahmed Abdullah Al Nasser ${ }^{4}$ \\ ${ }^{1}$ Periodontics and Community Dental Sciences Department, College of Dentistry, King Khalid University, Abha, Saudi Arabia \\ ${ }^{2}$ Ministry of Health, King Khalid Hospital, Alkhaldiyah Primary Health Care Center, Tabuk, Saudi Arabia \\ ${ }^{3}$ Ministry of Health, Al Qahmah Hospital, Asir, Saudi Arabia \\ ${ }^{4}$ Ministry of Health, Mnshabah Primary Health Care Center, Jazan, Saudi Arabia \\ Email: ^malabdaly20@gmail.com
}

How to cite this paper: Al-Abdaly, M.M.A.A., Alghamdi, A.S., Alshehri, A.H. and Al Nasser, F.A.A. (2021) Topical Application of Ascorbic Acid Solution as an Adjunctive Method of Periodontal Therapy among Wet Snuff Users after Prosthodontics Therapy. Open Journal of Stomatology, 11, 119-132.

https://doi.org/10.4236/ojst.2021.113010

Received: February 5, 2021

Accepted: March 12, 2021

Published: March 15, 2021

Copyright $\odot 2021$ by author(s) and Scientific Research Publishing Inc. This work is licensed under the Creative Commons Attribution International License (CC BY 4.0).

http://creativecommons.org/licenses/by/4.0/

cc) (i) Open Access

\begin{abstract}
Background: During several years, ascorbic acid (vitamin C) played a significant role in the health of periodontal tissues. The goals of prosthodontics management are to restore the missing teeth, provide the loss of teeth functions, and establish acceptable dental esthetics, and there are some evidences revealing the passive effects of snuff on the results of prosthodontics and periodontal treatment, so the present study is performed to evaluate the outcomes of topical application of ascorbic acid solution as an adjunctive method of periodontal therapy among wet sniff users after prosthodontics therapy. Methods: The clinical situation was evaluated in 150 moderate periodontitis patients after conventional periodontal and prosthodontics therapy. They were selected from outpatient clinics, college of dentistry, King Khalid University, and from some hospitals Ministry of Health in Tabuk, Jazan and Aseer regions. The study was conducted from September 2020 to December 2020. The time of post-treatment assessment was one year. The study was included 50 patients who don't use wet snuff as a group I (GI) (control group), 50 patients wet snuff users as group II (GII), and 50 patients wet snuff users were treated with topical application of ascorbic acid solution as group III (GIII). The participants were aged over 20 years, with a mean age of 35 years. The duration of using wet snuff was at least one year. Plaque index (PLI), gingival index (GI), clinical attachment loss (CAL) were recorded at baseline (first visit), then after 4 weeks (second visit) and after 6 weeks (third visit) of
\end{abstract}


conventional periodontal therapy and topical application of ascorbic acid solution. Statistical analysis was done using ANOVA test and paired t-test. Results: According to statistical analysis, there was the inclination of decrease in the mean and standard deviation of plaque index (PLI) from $2.8 \pm 1.4$ to $2.5 \pm$ 0.51 , and $2.7 \pm 0.33$ in group II, and decrease from $2.7 \pm 0.34$ to $2.5 \pm 0.23$ and $2.6 \pm 0.65$ in group III. Similarly in the gingival index (GI), there was a decrease in the mean and standard deviation from $2.8 \pm 0.52$ to $2.3 \pm 0.62$ and $2.5 \pm 0.51$ in group II and a decrease from $2.5 \pm 0.8$ to $2.3 \pm 0.58$ and $2.4 \pm$ 0.47 in group III. Moreover, there was a decrease in the mean and standard deviation of clinical attachment loss (CAL) from $4.5 \pm 0.26$ to $3.9 \pm 0.83$ and $4.2 \pm 0.83$ in group II and from $4.2 \pm 0.31$ to $3.8 \pm 0.46$ and $3.8 \pm 0.31$ in group III; less than group I without significance differences $(\mathrm{p}>0.05)$ in all study groups at baseline and after 4 weeks, and 6 weeks of follow up except CAL. Conclusion: The results of our study displayed that periodontal parameters vary among wet snuff users after topical application of ascorbic acid solution as an adjunctive method of periodontal therapy when compared to the patients who do not use snuff after conventional periodontal and prosthodontics therapy at baseline and after 4 weeks, and 6 weeks of follow up. Therefore, ascorbic acid solution can be used as an adjunctive and effective method in periodontal disease therapy among wet snuff users.

\section{Keywords}

An Adjunctive Periodontal Therapy, Ascorbic Acid Solution, Prosthodontics Therapy, Wet Snuff

\section{Introduction}

The dental prosthetic treatment has an effect on periodontal tissues that plays a significant role in the prognosis of periodontal therapy where prosthodontics appliances are considered as predisposing factors for progressing and recurrence of periodontal diseases postoperatively due to their passive effects on plaque accumulation, so the definitive assessment of prosthodontics treatment must not depend on the function and aesthetic standards only, but also depend on their impacts on periodontal tissues [1].

Understanding how the prosthodontics appliances participate in the accumulation of dental plaque and periodontal diseases is very important. The improper design of the restoration margins extension may produce harmful effects on the gingival sulcus. Furthermore, any defect in the designing of these margins may play a significant role in the development of periodontal diseases [2].

Periodontal disease is a mixed infection that occurs as complex interactions between specific bacteria, hereditary, and ecological factors especially, tobacco consumption and it's characterized by excessive release of free radicals ions of oxygen by chronic inflammatory cells, like neutrophils [3] [4].

The significance of ascorbic acid in the health of periodontium has been rec- 
ognized since many years ago, and it is an antioxidant agent against oxygen-free radical ions that can contribute to periodontal tissue destruction among periodontitis patients [5]. Moreover, conventional periodontal therapy has been the foundation for the control of dental plaque and periodontitis prevention [6].

Snuff is produced by grinding tobacco leaves, which can be used dry or wet. The dry type is a powder that is used by the nose, while the wet type is used by putting it between the cheek and lower lip or gingiva and cheek, facilitating nicotine absorption by the oral mucosa [7] [8]. Snuff use is considered from the environmental risk factors of increased spread and intensity of the periodontal disease, and the risk effects of snuff use on periodontal and prosthodontics therapy outcomes depend on the strengthened dose and years of exposure to tobacco products [9] [10] [11].

Few studies assessed the outcomes of periodontal therapy with and without snuff use, but there was no study on topical application of ascorbic acid solution as an adjunctive method of periodontal treatment with wet sniff using among Saudi people after prosthodontics therapy. Moreover, identify snuff use as an ecological risk factor of periodontal disease is still complicated due to the difficulty of evaluating the exact effect of tobacco products. Generally, there are relationships in the literature between snuff use and the severity of periodontal breakdown and the efficiency of periodontal therapy that is more efficient among the patients who do not use snuff more than snuff users [12] [13] [14] [15].

On the other hand, there is some evidence that the response to periodontal treatment depends on the amount of tobacco consumption, and based on the close and the inherent relation between prosthodontics appliances and periodontal therapy results, the present study performed to evaluate the effect of wet snuff use on the outcomes of periodontal therapy among Saudi people who received prosthodontics therapy [16]. This study hypothesized that prosthodontics appliances after periodontal therapy do not preserve periodontal tissues among wet snuff users patients due to their impact on dental plaque formation and self plaque control. Therefore, this study was designed to evaluate the topical application of ascorbic acid solution as an adjunctive method of periodontal therapy among wet snuff users after prosthodontics therapy.

\section{Subjects and Methods}

\subsection{Sample Size and Ethics}

As sample size, one hundred and fifty patients over the age of 20 years old selected after periodontal and prosthodontics therapy from outpatient clinics, college of dentistry, King Khalid University, and from some hospitals Ministry of Health in Tabuk, Jazan, and Aseer regions from September 2020 to December 2020 , one year is the time of post-treatment assessment. The study was designed according to the instruction of the research ethics committee, college of denti-

stry, King Khalid University. The informed consent of patients obtained before they participated in the study. 


\subsection{Study Design}

\subsubsection{Inclusion Criteria}

The patients divided into three equal groups of 50 participants each, treated by conventional periodontal therapy after prosthodontics therapy. Group I (control group): It included patients who do not use wet snuff. Group II: Wet snuff users. Group III: Wet snuff users treated with topical application of ascorbic acid solution, at least one year is the duration of wet snuff use, at least one time per day.

\subsubsection{Exclusion Criteria}

The case history obtained to exclude:

- The patients with systemic diseases.

- Cigarette smoking patients.

- Menopause patients,

- Tobacco chewing patients.

- Pregnant patients.

- The patients who received anti-inflammatory/antibiotic, corticosteroid therapy, or periodontal therapy in the six months before the study.

- The patients who usage of vitamin supplements and antioxidants supplement intake or, the patients who regularly use of mouthwash in the three months before the study, and the lactating women [17].

\subsubsection{Clinical History}

The clinical case history of patients was recorded and including age, address, gender, occupation, systemic conditions, medications, and details about snuff using (frequency and duration) [18].

\subsubsection{Clinical Examination}

A complete periodontal examination performed, and the following indices recorded by using a Williams periodontal probe: Plaque index (PLI) [19], gingival index (GI) [20], and clinical attachment loss (CAL) at baseline (first visit) and after 4 weeks (second visit), and 6 weeks (third visit) of the study intervals.

\subsubsection{Periodontal Therapy}

The oral hygiene instructions for self-plaque control were given to the patients before the start of periodontal therapy, then conventional periodontal treatment performed on all patients of group I, II, and III, and ascorbic acid solution (ampoules $250 \mathrm{mg} / \mathrm{mL}$ ) was used on group III participants, where it applied for five minutes into the periodontal pocket by using a blunt-ended cannula after isolation of the area, then the patient was requested not to wash the mouth and not eat at least thirty minutes of the procedure. The clinical findings collected in the first visit and second visit, and third visit.

\subsection{Statistical Analysis}

The statistical analyses of the collected data were done. ANOVA test was used to know the mean of participants' age and its distribution according to their gend- 
er. Moreover, it was used to detect the significant differences of periodontal parameters in the participants' visits within the group. The paired t-test was used to detect the significant differences of periodontal parameters between the groups in the first visit, second visit, and third visit after periodontal therapy. Data were exhibited as mean and standard deviation $( \pm S D)$. The statistically significant differences were considered when $\mathrm{P}$-value was less than $0.05(\mathrm{P}<0.05)$.

\section{Results}

One hundred and fifty participants were evaluated, aged $20-60$ years. There were 100 urban Saudi participants with a mean age of $38.2 \pm 6.4$ years and 50 rural Saudi participants with a mean age of $45.7 \pm 9.7$ years. There was a difference in age between urban Saudi and rural Saudi participants without statistical significance differences $(\mathrm{P}=0.401)$ (Table 1 and Figure 1$)$. The assessment of the collected data of this study clarified that the wet snuff use was not associated with the living area of urban and rural Saudi participants.

The clinical parameters' assessment of the study group in the first visit, the second visit, and the third visit was summarized in Table 2 and Figure 2. There were detected differences in the clinical parameters without statistically significant differences during the comparison between the first visit, second visit, and third visit of periodontal therapy among all participants except CAL $(\mathrm{P}>0.05)$. There was an increase in all clinical parameters among the patients of group II

Table 1. Participants age distribution.

\begin{tabular}{ccccc}
\hline & Rage of age & Mean \pm SD & \multicolumn{2}{c}{ ANOVA } \\
\cline { 4 - 5 } & & & F & P-value \\
\hline USP & $20-50$ & $38.2 \pm 6.4$ & 2.13 & 0.401 \\
RSP & $25-60$ & $45.7 \pm 9.7$ & & \\
\hline
\end{tabular}

USP: Urban Saudi Participants. RSP: Rural Saudi Participants.

Table 2. Comparison between the study groups in clinical parameters.

\begin{tabular}{ccccccc}
\hline \multirow{2}{*}{ SG } & CF & Base line & After 4 W & After 6 W & \multicolumn{2}{c}{ ANOVA } \\
\cline { 6 - 7 } & & & & F & P-Value \\
\hline \multirow{2}{*}{ Group I } & GLI & $1.8 \pm 0.21$ & $1.4 \pm 0.11$ & $1.4 \pm 0.43$ & 0.812 & 0.086 \\
& GI & $2.1 \pm 0.3$ & $1.4 \pm 0.41$ & $1.4 \pm 0.41$ & 0.025 & 0.062 \\
& CAL & $3.5 \pm 0.36$ & $2.5 \pm 0.41$ & $1.7 \pm 0.37$ & 0.031 & $0.031^{*}$ \\
Group II & GLI & $2.8 \pm 1.4$ & $2.5 \pm 0.51$ & $2.7 \pm 0.33$ & 0.411 & 0.723 \\
& GI & $2.8 \pm 0.52$ & $2.3 \pm 0.62$ & $2.5 \pm 0.51$ & 0.053 & 0.767 \\
& CAL & $4.5 \pm 0.26$ & $3.9 \pm 0.83$ & $4.2 \pm 0.83$ & 0.427 & 0.481 \\
Group III & GI & $2.7 \pm 0.34$ & $2.5 \pm 0.23$ & $2.6 \pm 0.65$ & 0.154 & 0.870 \\
& CAL & $4.2 \pm 0.31$ & $3.8 \pm 0.46$ & $3.8 \pm 0.31$ & 0.165 & 0.651 \\
\hline
\end{tabular}

SG: Study group. CF: Clinical findings. W: Week PLI: Plaque index. GI: Gingival index. CAL: Clinical attachment loss. ${ }^{*}$ Statistical significant differences $(\mathrm{P}<0.05)$. 


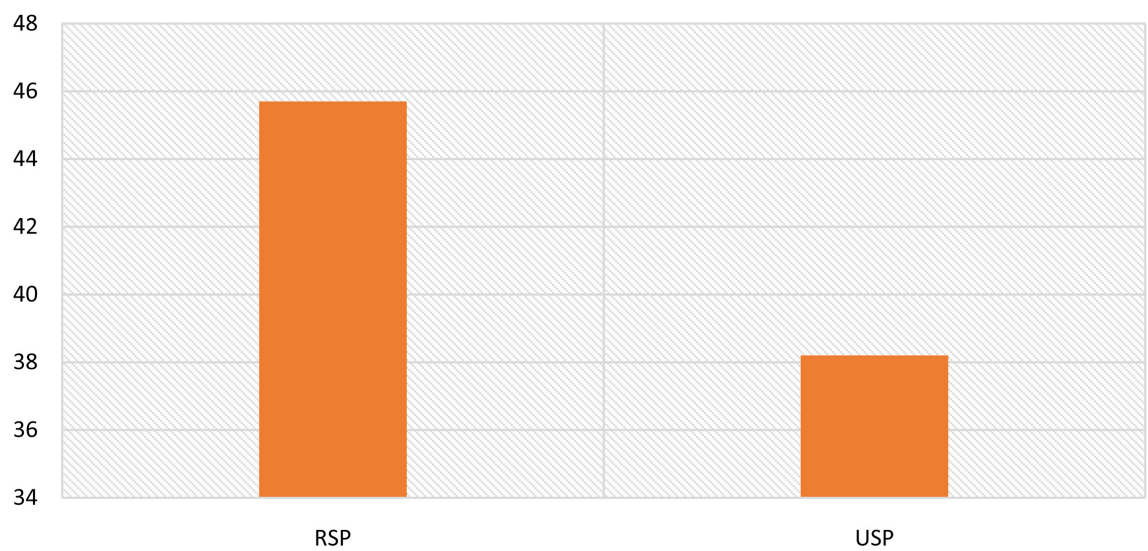

Figure 1. Age participants distribution. USP: Urban Saudi Participants. RSP: Rural Saudi Participant.

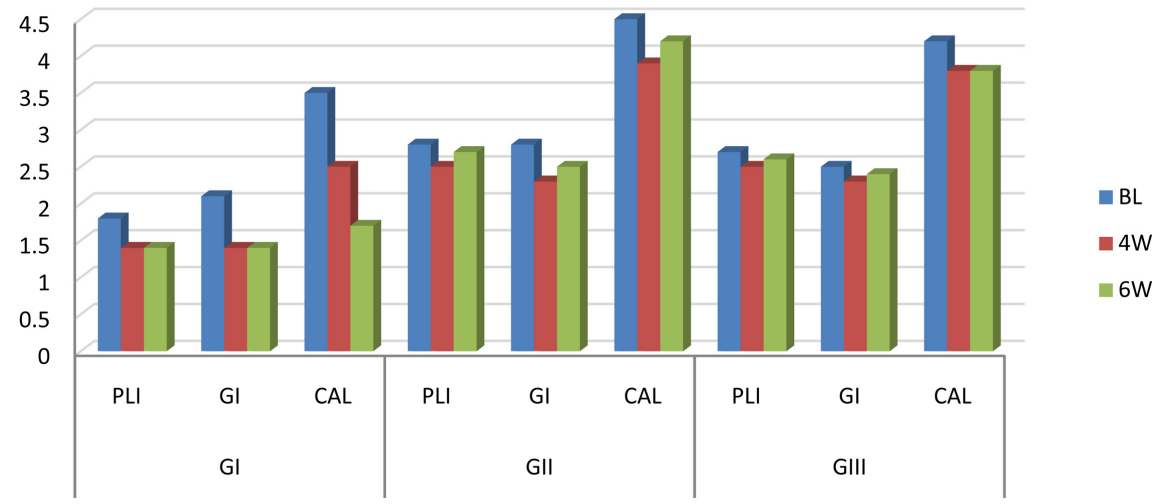

Figure 2. Comparison between the study groups in clinical parameters. SG: Study group. W: Week PLI: Plaque index. GI: Gingival index. CAL: Clinical attachment loss.

and group III more than group I. More decrease was observed in all clinical parameters for the group of participants, who were not wet snuff users after 4 weeks, and 6 weeks follow-up. Table 2 and Figure 2 showed better results of group I who were not wet snuff users and treated with conventional therapy alone as compared to wet snuff users in group II and group III, but the reduction was more among the participants in group III who treated with topical application of ascorbic acid solution than the participants in group II who treated with conventional periodontal therapy alone.

Consequently, there was a reduction in the mean and standard deviation of plaque index (PLI) from $2.8 \pm 1.4$ to $2.5 \pm 0.51$, and $2.7 \pm 0.33$ in group II, and a reduction from $2.7 \pm 0.34$ to $2.5 \pm 0.23$ and $2.6 \pm 0.65$ in group III. Consistent with clinical assessment of gingival status where there was a reduction in the mean and standard deviation of the gingival index (GI) from $2.8 \pm 0.52$ to $2.3 \pm$ 0.62 and $2.5 \pm 0.51$ in group II and a reduction from $2.5 \pm 0.8$ to $2.3 \pm 0.58$ and $2.4 \pm 0.47$ in group III. Moreover, there was a reduction in the mean and standard deviation of clinical attachment loss (CAL) from $4.5 \pm 0.26$ to $3.9 \pm 0.83$ and $4.2 \pm 0.83$ in group II and from $4.2 \pm 0.31$ to $3.8 \pm 0.46$ and $3.8 \pm 0.31$ in group III; as compared with group I without significance differences $(\mathrm{P}>0.05)$ 
in all study groups at baseline and after 4 weeks, and 6 weeks of follow up except CAL.

On the other hand, there were differences of periodontal parameters in the comparison between the first visit and the second visit, and between the first visit and the third visit and between the second visit and the third visit without statistical significance differences except for plaque index, gingival index, and CAL in the comparison between the first visit and the second visit follow up, and between first visit and the third visit among the patients of group I and CAL among the patients of group II moreover CAL in the comparison between the second visit and the third visit where there were statistical significance differences $(\mathrm{P}<0.05)$ (Table 3 ).

There was a decrease in all clinical parameters in the second visit and the third visit amongst all participants compared to the first visit. This decrease was more among the patients who do not use wet snuff and received conventional periodontal therapy after prosthodontics therapy compared to wet snuff users patients and received conventional periodontal therapy after prosthodontics therapy and wet snuff users patients received conventional periodontal therapy with topical application of ascorbic acid solution. Consequently, wet snuff use with

Table 3. Comparison between clinical parameters within the study groups at baseline, 4 months and 6 months.

\begin{tabular}{|c|c|c|c|c|c|c|c|c|c|c|}
\hline \multirow{3}{*}{ SG } & \multirow{3}{*}{$\mathrm{CF}$} & \multirow{3}{*}{$\mathrm{BL}$} & \multirow{3}{*}{$4 \mathrm{~W}$} & \multirow{3}{*}{$6 \mathrm{~W}$} & \multicolumn{6}{|c|}{$\mathrm{T}$ - test } \\
\hline & & & & & \multicolumn{2}{|c|}{ BL-4M } & \multicolumn{2}{|c|}{ BL-6M } & \multicolumn{2}{|c|}{$4 \mathrm{M}-6 \mathrm{M}$} \\
\hline & & & & & $\mathrm{T}$ & $\mathrm{P}-\mathrm{v}$ & $\mathrm{T}$ & P-v & $\mathrm{T}$ & $\mathrm{P}-\mathrm{v}$ \\
\hline \multirow{3}{*}{ PLI } & GI & $1.8 \pm 0.21$ & $1.4 \pm 0.11$ & $1.4 \pm 0.43$ & 2.967 & $0.010^{*}$ & 1.855 & $0.018^{*}$ & 1.203 & 0.407 \\
\hline & GII & $2.8 \pm 1.4$ & $2.5 \pm 0.51$ & $2.7 \pm 0.33$ & 1.433 & 0.195 & 2.236 & 0.285 & 1.321 & 0.161 \\
\hline & GIII & $2.7 \pm 0.34$ & $2.5 \pm 0.23$ & $2.6 \pm 0.65$ & 1.333 & 0.125 & 1.153 & 0.164 & 1.651 & 0.336 \\
\hline \multirow{2}{*}{ T-test } & $\mathrm{T}$ & 3.000 & 1.203 & -2.00 & & & & & & \\
\hline & P-v & 0.124 & 0.183 & $0.022^{*}$ & & & & & & \\
\hline \multirow{3}{*}{ GI } & GI & $2.1 \pm 0.3$ & $1.4 \pm 0.41$ & $1.4 \pm 0.41$ & 3.000 & $<0.05^{*}$ & 3.000 & $<0.05^{\star}$ & 1.185 & 0.184 \\
\hline & GII & $2.8 \pm 0.52$ & $2.3 \pm 0.62$ & $2.5 \pm 0.51$ & 0.279 & 0.256 & 0.139 & 0.148 & 0.146 & 0.159 \\
\hline & GIII & $2.5 \pm 0.8$ & $2.3 \pm 0.58$ & $2.4 \pm 0.47$ & 0.585 & 0.179 & 0.546 & 0.173 & 0.519 & 0.181 \\
\hline \multirow{2}{*}{ T-test } & $\mathrm{T}$ & 1.123 & 2.981 & -1.873 & & & & & & \\
\hline & $\mathrm{P}-\mathrm{v}$ & 0.243 & 0.072 & $0.051^{*}$ & & & & & & \\
\hline \multirow{3}{*}{ CAL } & GI & $3.5 \pm 0.36$ & $2.5 \pm 0.41$ & $1.7 \pm 0.37$ & 2.973 & $0.013^{*}$ & 2.385 & $0.01^{*}$ & 1.300 & $0.008^{*}$ \\
\hline & GII & $4.5 \pm 0.26$ & $3.9 \pm 0.83$ & $4.2 \pm 0.83$ & 2.371 & $0.05^{\star}$ & 5.401 & $0.05^{\star}$ & 1.314 & 0.149 \\
\hline & GIII & $4.2 \pm 0.31$ & $3.8 \pm 0.46$ & $3.8 \pm 0.31$ & 5.721 & 0.067 & 3.341 & 0.074 & 1.704 & 0.092 \\
\hline \multirow{2}{*}{ T-test } & $\mathrm{T}$ & 2.140 & 4.860 & 1.400 & & & & & & \\
\hline & $\mathrm{P}-\mathrm{v}$ & $0.034^{*}$ & $0.014^{*}$ & $0.022^{*}$ & & & & & & \\
\hline
\end{tabular}

GI: group I. GII: group II. GIII: group III. BL: Baseline. CF: Clinical finding. W: Week PLI: Plaque index. GI: Gingival index. CAL: Clinical attachment loss. ${ }^{\star}$ Statistical significant differences $(\mathrm{P}<0.05)$. 


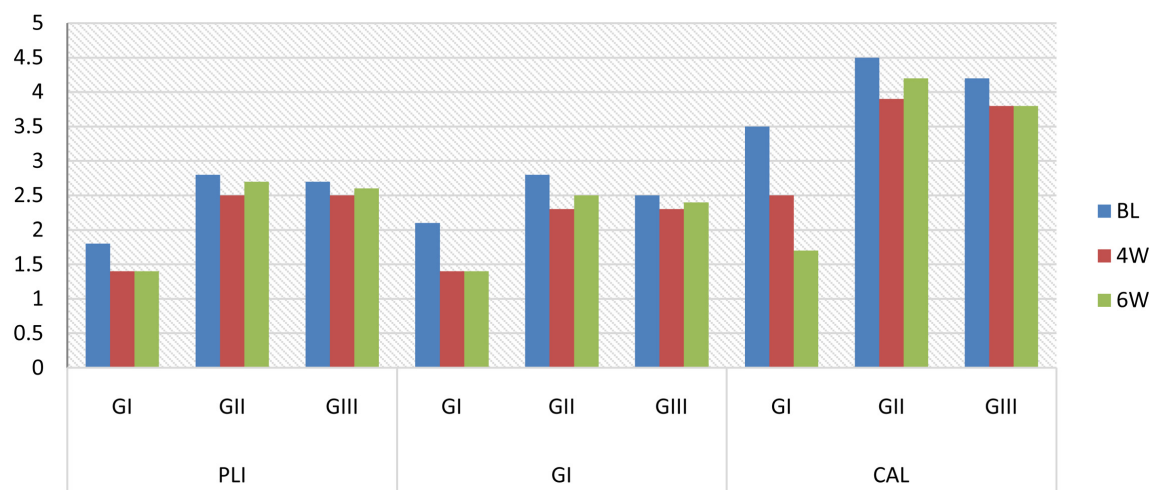

Figure 3. Comparison between clinical parameters within study groups. GI: group I. GII: group II. GIII: group III. BL: Baseline. W: Week PLI: Plaque index. GI: Gingival index. CAL: Clinical attachment loss.

Table 4. The matrix correlation between age, plaque index, gingival index and CAL.

\begin{tabular}{ccccc}
\hline & & Age & PLI & GI \\
\hline \multirow{2}{*}{ PLI } & r & -0.148 & & \\
& P-value & 0.301 & & \\
GI & r & 0.173 & 0.215 & \\
& P-value & 0.221 & 0.078 & 0.051 \\
CAL & r & -0.110 & -0.087 & 0.634 \\
& P-value & 0.413 & 0.506 & \\
\hline
\end{tabular}

PLI: Plaque index. GI: Gingival index. CAL: Clinical attachment loss.

prosthodontics appliances had the maximum impact on periodontal therapy outcomes more than wet snuff use alone and is meaning a less appropriate response to periodontal therapy among this group's participants (Table 3 and Figure 3). According to the statistical analysis in Table 4, there was no matrix correlation between Age, Plaque Index, Gingival Index, and clinical attachment loss in the current study.

\section{Dissection}

Wet snuff is a tobacco product that used with placed into the mouth between the cheek and the lip, and consequently, affect periodontal tissues where there was an increase in periodontal pockets among wet snuff users more than non-wet snuff users, as we found in the present study where there was more effect of wet snuff using on the clinical parameters of periodontal status assessment [21]. Moreover, the ability of prosthodontics appliances to catch dental plaque is a risk factor, and elucidates the relation between the increase of periodontal diseases on the abutment and the partial dentures [22] [23]. Ascorbic Acid is an antioxidant can decrease the generation of reactive oxygen species that are associated with inflammatory reaction in periodontal diseases, and cause more destruction of periodontal tissues [24] [25]. 
The design of this study was based on the clinical evaluation of topical application effectiveness of ascorbic acid solution in periodontal therapy of wet snuff users after prosthodontics therapy and the relationship between the type periodontal therapy and the improvement of periodontitis after 4 weeks and 6 weeks follow up and using of wet snuff as compared with non-using of wet snuff.

The range of age in this study was 20 - 60 years. It represents the effects of wet snuff use on periodontal tissues among adults and older adults. Similar observations of Kumar et al. where observed an increase in smokeless tobacco using after 21 years old [26]. There was no correlation between the living area of participants and wet snuff using in the current study. In agreement with another study that exhibited, there were no significant differences in smoking between rural and urban areas [27].

Our study demonstrated a passive correlation between plaque index, gingival index, and clinical attachment loss with the use of wet snuff after periodontal therapy, as was clarified by Hirsch et al. [28] and Grady et al. [29].

Smokeless tobacco may be a predisposing factor for loss of attachment in the maintenance phase of periodontal therapy with patients who susceptible to periodontitis due to affect some ecological, genetic, and host factors [30], and according to a recent study, there was an increase in the intensity of periodontal tissue destruction and minimal response to non-surgical periodontal therapy during tobacco consumption compared to do not tobacco consumption [31], and there was a correlation between consumption of tobacco and accumulation of dental plaque on teeth surfaces [32], which is in agreement with the clinical findings of the present study where there was an increase in periodontal destruction among wet snuff users (group II and III) more than patients who do not use wet snuff (group I). Furthermore, there was a reduction in response to conventional periodontal therapy and topical application of ascorbic acid solution in all visits of follow-up.

On the other hand and according to the results of another study, there was less improvement in clinical parameters with consumption of tobacco as compared with do not tobacco consumption in the 6- to 8-week after scaling and root planning, so we should know that the consumption of tobacco has passive effects on both conventional periodontal therapy and periodontal therapy with chemotherapeutic agents and this, is agreed, with the clinical finding of the present study where the results revealed that GII and GIII had PLI, GI and CAL more than GI (control group) at baseline and 4, and 6 weeks of the study intervals [33].

Clinical findings in baseline visits (first visit) are more important when comparing the response to treatment among two or more study groups in different follow-up visits. The current study groups exhibited similar clinical findings in the baseline visit of oral health status, inflammation of gingival tissues, and destructive periodontal diseases.

After mechanical periodontal therapy alone and with topical application of 
ascorbic acid solution as an adjunctive method of periodontal therapy, the measurement of clinical periodontal parameters appeared the decreased similarly in both the patients who use wet snuff and the patients who do not use wet snuff. These clinical findings are harmonious with a study conducted by De Genaro Modanese et al. indicated a similar decrease in PLI and GI and CAL in the smoker and non-smoker groups between baseline and 6months after mechanical periodontal therapy [34].

Some studies detected that the application of some chemotherapeutic agents in the treatment of some types of periodontal diseases show limited improvements, consistent with the clinical findings of this study that certified there were slight improvement in clinical results of the patients treated with both mechanical periodontal therapy, and topical application of ascorbic acid solution than the clinical findings of the patients was treated with conventional periodontal treatment alone at the second and third visit as compared to the first visit [35] [36] [37]. These clinical findings agree with the study results of Yussif NM, et al. who reported, that there were improvements in the inflamed gingival tissue after topical application of vitamin C [38]. The reduction of plaque index in the second visit and third visit as compared with the first visit may be attributed to the effectiveness of the oral hygiene measures by the patients.

Despite we conducted the conventional periodontal therapy of all patients and topical application of ascorbic acid solution of the patients in group III. The gingival index indicates that there is moderate gingivitis with the patients who do not use wet snuff and severe gingivitis among the wet snuff users and wet snuff users who were treated with topical application of ascorbic acid solution at baseline (first visit) and after 4 weeks (second visit), and 6 weeks (third visit) of the study intervals. Moreover, plaque index shows that there is moderate plaque accumulation/moderate oral hygiene of the patients who do not use wet snuff and heavy plaque accumulation/poor oral hygiene among the wet snuff users and wet snuff users who were treated with topical application of ascorbic acid solution at baseline (first visit) and after 4 weeks (second visit), and 6 weeks (third visit) of the study intervals.

Finally, clinical attachment loss shows that there is a change of periodontitis from moderate to mild of the patients who do not use wet snuff, and there is a change from severe to moderate among the wet snuff users and wet snuff users who were treated with topical application of ascorbic acid solution at baseline (first visit) and after 4 weeks (second visit) and 6 weeks (third visit) of the study intervals. These clinical findings are identical to the study conducted by Yong $\mathrm{H}$. Chu et al. using the same clinical parameters [39].

\section{Strength and Limitations}

The topical application of ascorbic acid solution was chosen in the present study rather than the topical dentifrice or gel to increase its superficial penetrating effect. 
There were many limitations in the current study, such as the limited absorption of ascorbic acid, its ease of removal from oral mucosa when exposed to saliva. The improvement in the periodontal status led to increasing cooperation of the patients and enhanced their contentment to the end of the study. Therefore, we advise applying ascorbic acid solution injection in the periodontal pocket as an adjunctive method for treatment of periodontitis.

\section{Conclusion}

Despite the limitation of this study, it may be concluded that wet snuff users who are treated with both conventional periodontal therapy and topical application of ascorbic acid solution exhibit more improvement of destructive periodontal diseases. We suggest conducting additional studies include lab investigations with long follow-up durations, and moreover, using other topical application of chemotherapeutic agents in treatment of periodontal diseases among wet snuff users to develop the results of the present study.

\section{Acknowledgements}

The authors want to thank the dental students, interns, and staff members in the college of dentistry clinics, King Khalid University for, their beneficial helping.

\section{Conflicts of Interest}

There are no conflicts of interest.

\section{References}

[1] Giollo, M.D., Valle, P.M., Gomes, S.C. and Rösing, C.K. (2007) A Retrospective Clinical, Radiographic and Microbiological Study of Periodontal Conditions of Teeth with and without Crowns. Brazilian Oral Research, 21, 348-354. https://doi.org/10.1590/S1806-83242007000400012

[2] Kwok, V. and Caton, J.G. (2007) Commentary: Prognosis Revisited: A System for Assigning Periodontal Prognosis. Journal of Periodontology, 78, 2063-2071. https://doi.org/10.1902/jop.2007.070210

[3] Labriola, A., Needleman, I. and Moles, D.R. (2005) Systematic Review of the Effect of Smoking on Nonsurgical Periodontal Therapy. Periodontology2000, 37, 124-137. https://doi.org/10.1111/j.1600-0757.2004.03793.x

[4] Chapple, I.L. (1997) Reactive Oxygen Species and Antioxidants in Inflammatory Diseases. Journal of Clinical Periodontology, 24, 287-296. https://doi.org/10.1111/j.1600-051X.1997.tb00760.x

[5] Wilson, J.X. (2005) Regulation of Vitamin C Transport. Annual Review of Nutrition, 25, 105-125. https://doi.org/10.1146/annurev.nutr.25.050304.092647

[6] Ongoz Dede, F., Bozkurt Dogan, S., Balli, U., Avci, B., Durmuşlar, M.C. and Baratzade, T. (2016) Glutathione Levels in Plasma, Saliva and Gingival Crevicular Fluid after Periodontal Therapy in Obese and Normal Weight Individuals. Journal of Periodontal Research, 51, 726-734. https://doi.org/10.1111/jre.12349

[7] Montén, U., Wennström, J.L. and Ramberg, P. (2006) Periodontal Conditions in Male Adolescents Using Smokeless Tobacco (Moist Snuff). Journal of Clinical Pe- 
riodontology, 33, 863-868. https://doi.org/10.1111/j.1600-051X.2006.01005.x

[8] Bergström, J., Keilani, H., Lundholm, C. and Rådestad, U. (2006) Smokeless Tobacco (Snuff) Use and Periodontal Bone Loss. Journal of Clinical Periodontology, 33, 549-554. https://doi.org/10.1111/j.1600-051X.2006.00945.X

[9] Haber, J., Wattles, J., Crowley, M., Mandell, R., Joshipura, K. and Kent, R.L. (1993) Evidence for Cigarette Smoking as a Major Risk Factor for Periodontitis. Journal of Periodontology, 64, 16-23. https://doi.org/10.1902/jop.1993.64.1.16

[10] Grossi, S.G., Genco, R.J., Machtei, E.E., Ho, A., Koch, G., Dunford, R., et al. (1995) Assessment of Risk for Periodontal Disease. II. Risk Indicators for Alveolar Bone Loss. Journal of Periodontology, 66, 23-29. https://doi.org/10.1902/jop.1995.66.1.23

[11] Hayman, L., Steffen, M.J., Stevens, J., Badger, E., Tempro, P., Fuller, B., et al. (2011) Smoking and Periodontal Disease: Discrimination of Antibody Responses to $\mathrm{Pa}$ thogenic and Commensal Oral Bacteria. Clinical \& Experimental Immunology, 164, 118-126. https://doi.org/10.1111/j.1365-2249.2010.04314.x

[12] Scott, D.A., Palmer, R.M. and Stapleton, J.A. (2001) Dose-Years as an Improved Index of Cumulative Tobacco Smoke Exposure. Medical Hypotheses, 56, 735-736. https://doi.org/10.1054/mehy.2001.1238

[13] Molloy, J., Wolff, L.F., Lopez-Guzman, A. and Hodges, J.S. (2004) The Association of Periodontal Disease Parameters with Systemic Medical Conditions and Tobacco Use. Journal of Clinical Periodontology, 31, 625-632. https://doi.org/10.1111/j.1600-051X.2004.00539.x

[14] Persson, R.E., Kiyak, A.H., Wyatt, C.C., MacEntee, M. and Persson, G.R. (2005) Smoking, a Weak Predictor of Periodontitis in Older Adults. Journal of Clinical Periodontology, 32, 512-517. https://doi.org/10.1111/j.1600-051X.2005.00701.x

[15] Kaldahl, W.B., Johnson, G.K., Patil, K.D. and Kalkwarf, K.L. (1996) Levels of Cigarette Consumption and Response to Periodontal Therapy. Journal of Periodontology, 67, 675-681. https://doi.org/10.1902/jop.1996.67.7.675

[16] Savita, M., Sarun, E., Arora, S. and Krishnan, S. (2015) Evaluation of Glutathione Level in Gingival Crevicular Fluid in Periodontal Health, in Chronic Periodontitis and after Nonsurgical Periodontal Therapy: A Clinicobiochemical Study. Contemporary Clinical Dentistry, 6, 206-210. https://doi.org/10.4103/0976-237X.156047

[17] Kosoko, A.M., Olayanju, O.A., Rahamon, S.K. and Arinola, O.G. (2017) Salivary Lipid Peroxidation and Antioxidant Status in Nigerian Cigarette Smokers with or without Periodontitis. Asian Journal of Medicine and Health, 3, 1-9. https://doi.org/10.9734/AJMAH/2017/30749

[18] Grossi, S.G., Zambon, J., Machtei, E.E., Schifferle, R., Andreana, S., Genco, R.J., et al. (1997) Effects of Smoking and Smoking Cessation on Healing after Mechanical Periodontal Therapy. Journal of the American Dental Association, 128, 599-607. https://doi.org/10.14219/jada.archive.1997.0259

[19] Silness, J. and Loe, H. (1964) Periodontal Disease in Pregnancy II. Correlation between Oral Hygiene and Periodontal Condition. Acta Odontologica Scandinavica, 22, 121-135. https://doi.org/10.3109/00016356408993968

[20] Loe, H. and Silness, J. (1963) Periodontal Disease in Pregnancy. I. Prevalence and Severity. Acta Odontologica Scandinavica, 21, 533-551.

https://doi.org/10.3109/00016356309011240

[21] Singh, G.P., Rizvi, I., Gupta, V. and Bains, V.K. (2011) Influence of Smokeless Tobacco on Periodontal Health Status in Local Population of North India: A Cross Sectional Study. Dental Research Journal, 8, 211-220. 
[22] Behr, M., Zeman, F., Passauer, T., Koller, M., Hahnel, S., Buergers, R., et al. (2012) Clinical Performance of Cast Clasp-Retained Removable Partial Dentures: A Retrospective Study. International Journal of Prosthodontics, 25, 138-144.

[23] Emami, E., Taraf, H., de Grandmont, P., Gauthier, G., de Koninck, L., Lamarche, C., et al. (2012) The Association of Denture Stomatitis and Partial Removable Dental Prostheses: A Systematic Review. International Journal of Prosthodontics, 25, 113-119.

[24] Chapple, I.L. (2009) Potential Mechanisms Underpinning the Nutritional Modulation of Periodontal Inflammation. Journal of the American Dental Association, 140, 178-184. https://doi.org/10.14219/jada.archive.2009.0131

[25] Wang, Y., Andrukhov, O. and Rausch-Fan, X. (2017) Oxidative Stress and Antioxidant System in Periodontitis. Frontiers in Physiology, 8, 910.

https://doi.org/10.3389/fphys.2017.00910

[26] Kumar, S., Prabu, D., Kulkarni, S. and Dagli, R.J. (2008) Tobacco as Risk Factor for Periodontal Disease in Green Marble Mine Laborers of Rajasthan, India. Brazilian Journal of Oral Sciences, 7, 1641-1647.

[27] Abdel Rahim, B.-E., Mahfouz, M.S., Yagoub, U., Solan, Y.M.H. and Alsanosy, R.M. (2014) Practice and Attitude of Cigarette Smoking: A Community-Based Study. PLOS ONE, 9, e92939. https://doi.org/10.1371/journal.pone.0092939

[28] Hirsch, J.M., Heyden, G. and Thilander, H. (1982) A Clinical, Histomorphological and Histochemical Study on Snuff-Induced Lesions. Journal of Oral Pathology \& Medicine, 11, 387-398. https://doi.org/10.1111/j.1600-0714.1982.tb00180.x

[29] Grady, D., Greene, J., Daniels, J.E., Ernster, V.L., Robertson, P.B., Hauck, W., et al. (1990) Oral Mucosal Lesions Found In Smokeless Tobacco Users. Journal of the American Dental Association, 121, 117-123. https://doi.org/10.14219/jada.archive.1990.0139

[30] Chambrone, L., Chambrone, D., Lima, L.A. and Chambrone, L.A. (2010) Predictors of Tooth Loss during Long-Term Periodontal Maintenance: A Systematic Review of observational Studies. Journal of Clinical Periodontology, 37, 675-684. https://doi.org/10.1111/j.1600-051X.2010.01587.x

[31] Goultschin, J., Cohen, H.D.S., Donchin, M., Brayer, L. and Aubrey Soskolne, W. (1990) Association of Smoking with Periodontal Treatment Needs. Journal of Periodontology, 61, 364-367. https://doi.org/10.1902/jop.1990.61.6.364

[32] Jansson, L.E. and Hagström, K.E. (2002) Relationship between Compliance and Periodontal Treatment Outcome in Smokers. Journal of Periodontology, 73, 602-607. https://doi.org/10.1902/jop.2002.73.6.602

[33] Darby, I.B., Hodge, P.J., Riggio, M.P. and Kinane, D.F. (2005) Clinical and Microbiological Effect of Scaling and Root Planing in Smoker and Non-Smoker Chronic and Aggressive Periodontitis Patients. Journal of Clinical Periodontology, 32, 200-206. https://doi.org/10.1111/j.1600-051X.2005.00644.x

[34] De Genaro Modanese, D., Tiosso-Tamburi, R., Furletti de Goes, V.F., de Cássia Bergamaschi, C., Martinez, E.F., Napimoga, M.H., et al. (2016) Clinical and Immunoinflammatory Evaluation of One-Stage Full-Mouth Ultrasonic Debridement as a Therapeutic Approach for Smokers with Generalized Aggressive Periodontitis: A Short-Term Follow-Up Study. Journal of Periodontology, 87, 1012-1021. https://doi.org/10.1902/jop.2016.150632

[35] Mestnik, M.J., Feres, M., Figueiredo, L.C., Duarte, P.M., Lira, E.A.G. and Faveri, M. (2010) Short-Term Benefits of the Adjunctive Use of Metronidazole Plus Amoxicillin in the Microbial Profile and in the Clinical Parameters of Subjects with Generalized Aggressive Periodontitis. Journal of Clinical Periodontology, 37, 353-365. 
https://doi.org/10.1111/j.1600-051X.2010.01538.x

[36] Keestra, J.A.J., Grosjean, I., Coucke, W., Quirynen, M. and Teughels, W. (2015) Non-Surgical Periodontal Therapy with Systemic Antibiotics in Patients with Untreated Aggressive Periodontitis: A Systematic Review and Meta-Analysis. Journal of Periodontal Research, 50, 689-706. https://doi.org/10.1111/jre.12252

[37] Varela, V.M., Heller, D., Silva-Senem, M.X., Torres, M.C.M.B., Colombo, A.P.V. and Feres-Filho, E.J. (2011) Systemic Antimicrobials Adjunctive to a Repeated Mechanical and Antiseptic Therapy for Aggressive Periodontitis: A 6 Month Randomized Controlled Trial. Journal of Periodontology, 82, 1121-1130. https://doi.org/10.1902/jop.2011.100656

[38] Yussif, N.M., Abdul Aziz, M.A. and Abdel Rahman, A.R. (2016) Evaluation of the Anti-Inflammatory Effect of Locally Delivered Vitamin C in the Treatment of Persistent Gingival Inflammation: Clinical and Histopathological Study. Journal of $\mathrm{Nu}$ trition and Metabolism, 2016, Article ID: 2978741.

https://doi.org/10.1155/2016/2978741

[39] Chu, Y.H., Tatakis, D.N. and Wee, A.G. (2010) Smokeless Tobacco Use and Periodontal Health in a Rural Male Population. Journal of Periodontology, 81, 848-854. https://doi.org/10.1902/jop.2010.090310 